\title{
Utility of Reticulocyte Parameters for the Diagnosis of Hereditary Spherocytosis
}

\author{
Ruchee Khanna ${ }^{1}$, Chethan Manohar ${ }^{2}$, Athira Sasidharan ${ }^{3}$ \\ ${ }^{1}$ Associate Professor, Department of Pathology, Kasturba Medical College, Manipal University, Manipal, Karnataka, \\ India. ${ }^{2}$ Professor, Department of Pathology, Kasturba Medical College, Manipal University, Manipal, Karnataka, India. \\ ${ }^{3}$ Assistant Professor, Department of Pathology, KMCT Medical College, Calicut, Kerala, India.
}

\section{ABSTRACT}

\section{BACKGROUND}

Hereditary spherocytosis is an inherited disorder of red blood cells which results in cytoskeleton protein deficiency. We wanted to assess the role of reticulocyte parameters in the diagnosis of hereditary spherocytosis.

\section{METHODS}

Cases in which osmotic fragility test was increased and peripheral smear showed spherocytes and polychromasia which were suggestive of hereditary spherocytosis were selected from Laboratory Information System (LIS). The study period was from January 2012 to December 2016.

\section{RESULTS}

The present study showed increase in retic \% and Red Cell Distribution Width (RDW) in cases of hereditary spherocytosis when compared to the normal controls and there was a significant statistical difference $(p<0.001)$, between the two groups. Area Under the Curve (AUC) was calculated for MCHC, IRF, MRV and Retic\#/IRF by using ROC curve analysis and was maximum for Retic\#/IRF (0.931), followed by MCHC (0.902), IRF (0.867) and MRV (0.865). Cut off values derived for RET\#/IRF, IRF, MRV and MCHC were ${ }^{3} 0.28,{ }^{3} 0.33, £ 98.8$ and ${ }^{3} 33.5$ respectively. Highest sensitivity and specificity was obtained for Ret\#/IRF which was $96 \%$ and $88 \%$ respectively. Percentage of cases with MRV $<$ MCV is $63.6 \%$.

\section{CONCLUSIONS}

The present study shows that reticulocyte parameters are useful in the diagnosis of Hereditary Spherocytosis. It proves that in addition to the presence of spherocytes in peripheral smear and an increased osmotic fragility test, reticulocyte parameters like RET\#/IRF ${ }^{3} 0.28, \mathrm{IRF}^{3} 0.33$ and MRV£ 98.8 are highly indicative of Hereditary Spherocytosis.

\section{KEY WORDS}

Retic Parameters, IRF, RET\#/IRF, MRV, Hereditary Spherocytosis
Corresponding Author: Dr. Athira Sasidharan, Anugraha, East Hill Road, Calicut-673006, Kerala, India. E-mail: athira87@gmail.com

DOI: $10.14260 / \mathrm{jemds} / 2019 / 630$

Financial or Other Competing Interests: None.

How to Cite This Article:

Khanna $R$, Manohar $C$, Sasidharan A. Utility of reticulocyte parameters for the diagnosis of hereditary spherocytosis. J. Evolution Med. Dent. Sci. 2019;8(38): 2902-2906, DOI:
Submission 18-07-2019,

Peer Review 05-09-2019,

Acceptance 12-09-2019,

Published 23-09-2019. 


\section{BACKGROUND}

Hereditary spherocytosis (HS) is a heterogenous group of inherited disorders affecting the red blood cells. They are characterized by structural membrane defects of the erythrocytes in turn affecting their deformability. It belongs to the group of congenital hemolytic anemias, named after the presence of spherocytes on peripheral blood smear. The incidence of hereditary spherocytosis in the Caucasian population is estimated to be about 1:2000 to 1: $5000 .^{1}$ The disease follows an autosomal dominant trait in about $70-75 \%$ of the cases. However, less commonly autosomal recessive pattern of inheritance is also seen. Rarely, de novo mutations in patients without a family history may also be seen.1,2

\section{Etiopathogenesis}

The deficiency in the various cytoskeletal membrane proteins forms the basis of hereditary spherocytosis. These defects decrease the deformability of the red cells and accelerate their degradation in the spleen. Most frequently affected are the genes encoding the membrane proteins Ankyrin, band3 and spectrin. ${ }^{2}$ The normal red cells are able to undergo passive deformations because of the maintenance of a favourable surface area to volume ratio. Passive deformations are essential so as to traverse the microvasculature for optimal oxygen delivery. Loss of cell surface area resulting in spherocytes becomes detrimental for red cell deformability and they are also rapidly sequestered from circulation by spleen. The surface area loss in HS which is due to the defect in the structural proteins is already present at the circulating reticulocyte stage, unlike in autoimmune hemolytic anemia. Progressive decrease in red cell surface area and cell volume occur during their life span in circulation. As a consequence of decreased cell volume, red cells with decreased membrane surface area have elevated cell haemoglobin concentration. ${ }^{2,3,4}$ HS reticulocytes from the very beginning have decreased cell volume and increased cell haemoglobin concentration, which probably result from an early attempt to counterbalance membrane loss. Consequently, it was hypothesised that automated reticulocyte parameters could be of great interest for hereditary spherocytosis screening. ${ }^{5}$

\section{Laboratory Diagnosis}

The diagnosis includes a meticulous medical and family history, along with laboratory parameters which include increased MCHC (Mean Corpuscular Hemoglobin Concentration) above the normal $(>35 \mathrm{~g} / \mathrm{dl})$, increased reticulocyte count along with biochemical parameters like increased LDH, indirect bilirubin and a negative coombs test. ${ }^{3}$ Osmotic fragility test may be employed as a screening test. Since the procedure was cumbersome and it required a high volume of blood, other tests were developed for the screening of HS. However, there is no single test for the diagnosis of HS with high sensitivity and specificity. The screening tests include osmotic fragility test, glycerol lysis test, Acidified glycerol Lysis Time (AGLT) test, Eosin 5-maleimide (EMA) binding test and cryohemolysis test. The confirmatory tests include membrane analysis by Sodium dodecyl sulfatepolyacrylamide gel electrophoresis (SDS PAGE), ektacytometry and genetic analysis.3,4 Improvement in the automated hematological analyzers have widened the spectrum of hematological parameters obtained from peripheral blood samples. The analysis of reticulocytes have evolved significantly. Analyzers now enumerate the reticulocyte volume, content of hemoglobin and differentiation into subpopulations of reticulocytes based on their stage of maturation. The reticulocyte parameters have been found to be a useful tool in detecting iron deficiency anemia and other types of anemias. 4,5

We wanted to evaluate the usefulness of reticulocyte parameters as a tool for diagnosis of hereditary spherocytosis.

\section{METHODS}

This is a retrospective cross-sectional study. A total of 25 cases of hereditary spherocytosis and an equal number of normal healthy controls were taken for this retrospective study conducted at Clinical Hematology and Clinical Pathology Laboratory, Department Of Pathology, Kasturba Medical College, Manipal. Sample size was taken based on the convenience of the study. The study period ranged from January 2012 to December 2016. The laboratory data were retrieved from the Laboratory Information System (LIS). The parameters retrieved were Osmotic fragility test reports, hematological parameters like hemoglobin, RBC count, Mean Cell Volume(MCV), Mean Corpuscular Hemoglobin Concentration (MCHC), Reticulocyte percentage, absolute reticulocyte count (Retic \#), Immature Reticulocyte Fraction (IRF), Mean Reticulocyte Volume (MRV) and Red cell Distribution width (RDW); along with reported peripheral smears. The study was approved by the Institute of Ethical Committee.

\section{Inclusion Criteria}

All the cases in which the peripheral smear findings were suggestive of Hereditary Spherocytosis and osmotic fragility test was increased.

\section{Exclusion Criteria}

Cases in which osmotic fragility was increased but did not correlate with the peripheral smear findings for the diagnosis of Hereditary Spherocytosis.

\section{Statistical Analysis}

Descriptive and group statistics. Tests used are t- test for equality of means. Area under the curve and thus Receiver Operated Curve were derived. Appropriate statistical analysis was carried out with Microsoft Excel 2013 and Statistical. Package for the Social Sciences (SPSS) version 20.0 (SPSS, Chicago, IL, USA).

\section{RESULTS}

Total number of cases of hereditary spherocytosis was 25 and Total number of normal healthy controls was 25 . 
The age range of patients with hereditary spherocytosis was 1 to 51 years, with a median age of 22 years. The haemoglobin concentration in 25 cases of hereditary spherocytosis ranged from $5.1-14.7 \mathrm{gm} / \mathrm{dl}$ with a mean haemoglobin concentration of $10.0 \mathrm{gm} / \mathrm{dl}$. (SD- 2.5) Considering the normal range of haemoglobin concentration to be between 12.0-15.0 gm/dl, (SD- 1.46) the present study showed that 18 out of 25 cases (72\%) had a haemoglobin concentration $<12 \mathrm{gm} / \mathrm{dl}$. (Table 1 )

Patients with Hereditary Spherocytosis showed increased reticulocyte percentage, Red Cell Distribution Width and Retic\#/IRF ratio when compared to that of normal subjects. The reticulocyte percentage was found to be significantly higher in patients with HS when compared to the normal healthy controls. (Table 2 and 3) The difference in reticulocyte $\%$ between patients with Hereditary spherocytosis and normal healthy controls was found to be statistically significant $(\mathrm{p}<0.001)$ (Fig. 1). The Red Cell Distribution Width (RDW) was found to be higher in patients with HS when compared to the normal healthy controls. (Table 4)

The difference in the mean Red Cell Distribution Width (RDW) between patients with Hereditary Spherocytosis and normal healthy controls was found to be statistically significant $(\mathrm{p}<0.001)$. The Absolute retic count was significantly higher in patients with HS when compared to the normal healthy controls. (Fig. 2) It was found that $63.6 \%$ of the cases with hereditary spherocytosis had MRV $<\mathrm{MCV}$, whereas in the normal controls all had an MRV greater than MCV (Fig. 4). According to the ROC curve analysis, the optimum value of MCHC was 33.55 giving a sensitivity of $86.4 \%$ and specificity of $80 \%$. According to the ROC curve analysis, the optimum value of MRV was $98.8 \mathrm{fl}$ giving a sensitivity of $92 \%$ and specificity of $80 \%$. According to the ROC curve analysis, the optimum value of IRF was 0.33 giving a sensitivity of $88 \%$ and specificity of $80 \%$. According to the ROC curve analysis, the optimum value of Retic\#/IRF was 0.28 giving a sensitivity of $96 \%$ and specificity of $88 \%$. It was found that the Area under Curve was maximum for Retic\#/IRF, followed by MCHC, IRF and MRV. Moreover, all these parameters were good predictors of the disease with the given cut-off values.

\begin{tabular}{|c|c|c|c|}
\hline Laboratory Parameter & Range & Median & S.D. \\
\hline Haemoglobin & $(5.1-14.7) \mathrm{gm} / \mathrm{dl}$ & $10 \mathrm{gm} / \mathrm{dl}$ & 2.5852 \\
\hline Red Blood Cell (RBC) Count & $(1.8-4.54) \times 10^{6} / \mathrm{ul}$ & $2.91 \times 10^{6} / \mathrm{ul}$ & .7567 \\
\hline Mean Cell Volume (MCV) & $(73.3-101.5) \mathrm{fl}$ & $87.75 \mathrm{fl}$ & 8.9457 \\
\hline $\begin{array}{c}\text { Mean Corpuscular Haemoglobin } \\
\text { Concentration (MCHC) }\end{array}$ & $(32.2-38.4) \mathrm{g} / \mathrm{dl}$ & $34.9 \mathrm{~g} / \mathrm{dl}$ & 1.4420 \\
\hline Retic \% & $(3.12-20.1) \%$ & $12.07 \%$ & 4.8303 \\
\hline Absolute Retic Count(Retic\#) & $(0.02-0.67)$ & 0.40 & .1648 \\
\hline Immature Reticulocyte Fraction (IRF) & $(0.22-0.70)$ & 0.43 & .1084 \\
\hline Mean Reticulocyte Volume (MRV) fl & $(65.4-135.5) \mathrm{fl}$ & $84.6 \mathrm{fl}$ & 16.8878 \\
\hline Red Cell Distribution Width (RDW) \% & $(13.6-27.5) \%$ & $19 \%$ & 3.3 \\
\hline Table 1. Haematological Parameters in Patients with Hereditary Spherocytosis \\
(n=25)
\end{tabular}

\begin{tabular}{|c|c|c|c|}
\hline Laboratory Parameter & Range & Median & S.D. \\
\hline Haemoglobin & $(12.0-18.4) \mathrm{gm} / \mathrm{dl}$ & $14.4 \mathrm{gm} / \mathrm{dl}$ & 2.5852 \\
\hline Red Blood Cell (RBC) Count & $(4.15-6.4) \times 10^{6} / \mathrm{ul}$ & $4.97 \times 10^{6} / \mathrm{ul}$ & .7567 \\
\hline Mean Cell Volume (MCV) & $(78.0-95.1) \mathrm{fl}$ & $87.0 \mathrm{fl}$ & 8.9457 \\
\hline $\begin{array}{c}\text { Mean Corpuscular Haemoglobin } \\
\text { Concentration (MCHC) }\end{array}$ & $(32.2-34.2) \mathrm{g} / \mathrm{dl}$ & $32.2 \mathrm{~g} / \mathrm{dl}$ & 1.4420 \\
\hline Retic\% & $(0.31-6.7) \%$ & $0.83 \%$ & 4.8303 \\
\hline Absolute Retic Count (Retic\#) & $(0.02-0.35)$ & 0.04 & .1648 \\
\hline Immature Reticulocyte Fraction (IRF) & $(0.20-0.50)$ & 0.30 & .1084 \\
\hline Mean ReticulocyteVolume (MRV) & $(94.2-121.2) \mathrm{fl}$ & $108.5 \mathrm{fl}$ & 16.8878 \\
\hline Red Cell DistributionWidth(RDW) & $(12.2-16.7) \%$ & $13.8 \%$ & 3.3 \\
\hline Table 2. Haematological Parameters in Normal Healthy Controls (n=25) \\
\hline
\end{tabular}

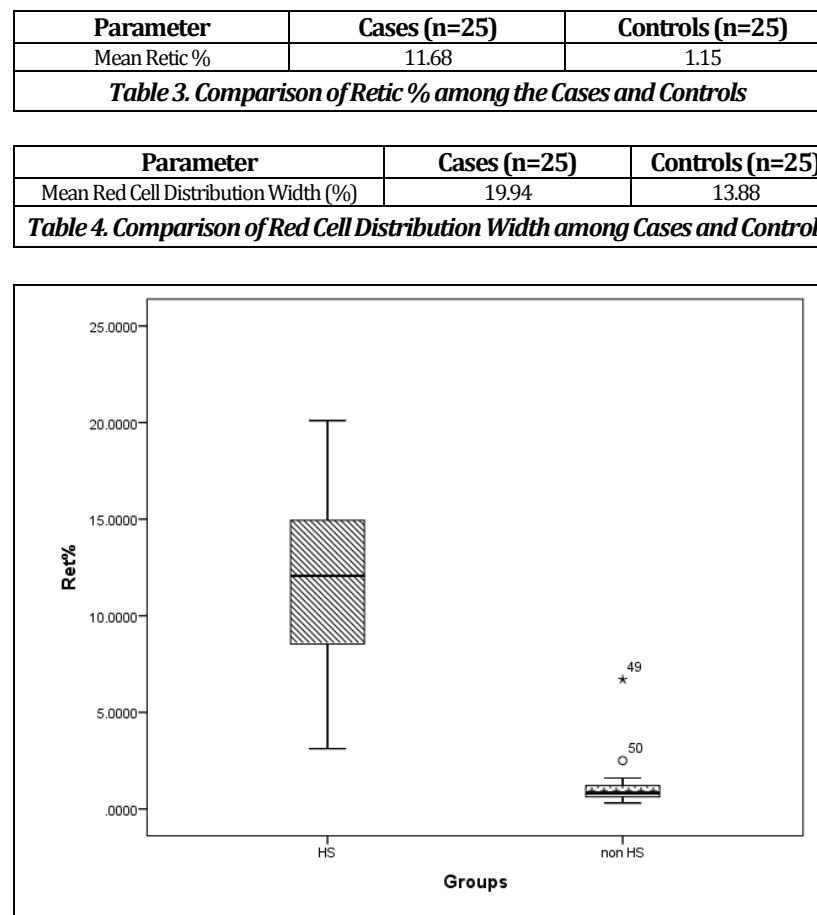

Figure 1. Comparison of Reticulocyte Percentage (Retic\%) between Patients with HS and those without HS

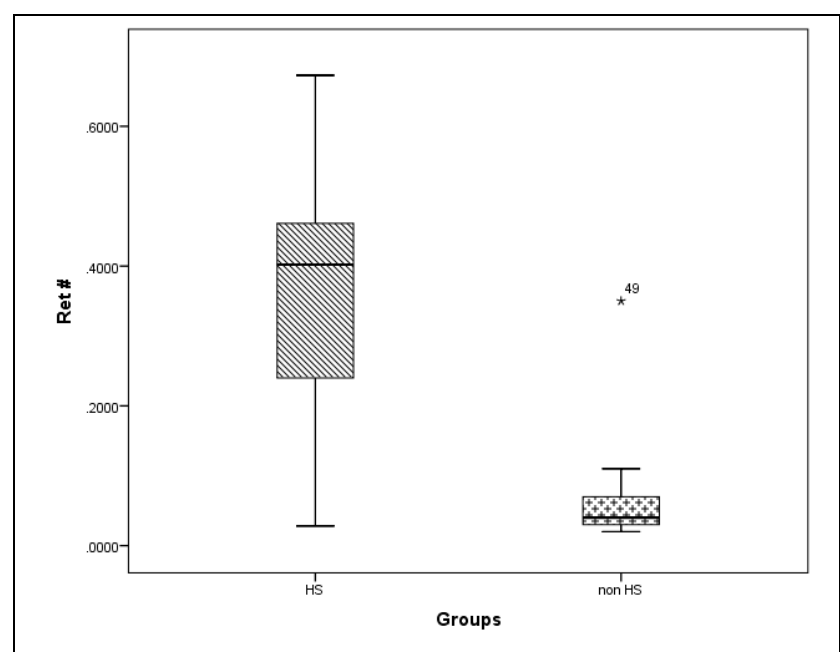

Figure 2. Comparison of Absolute Reticulocyte Count (retic\#) between Patients with HS and those without HS. Statistical Significance $* p<0.001$.

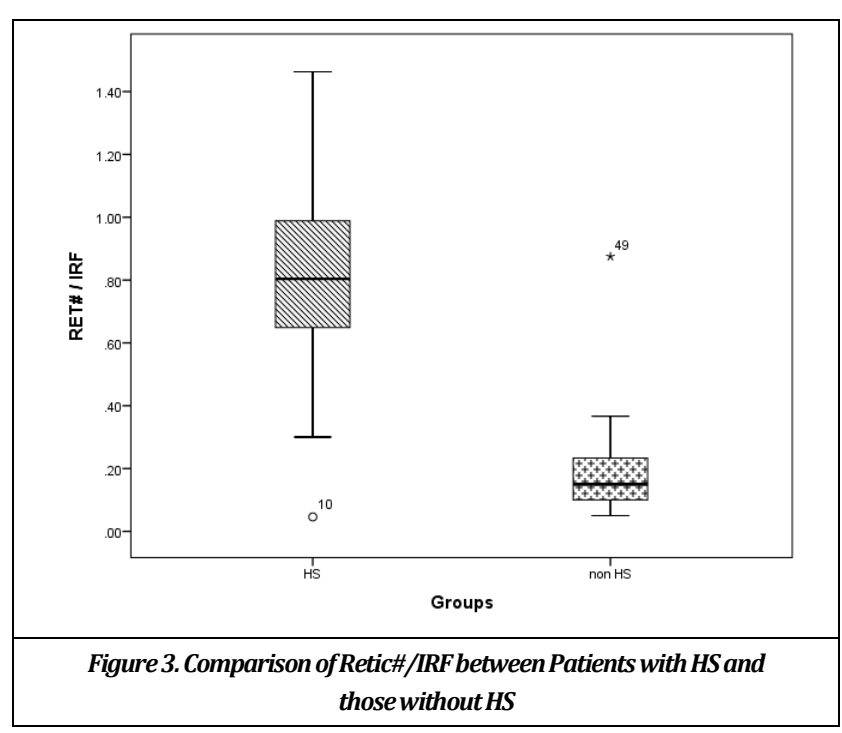




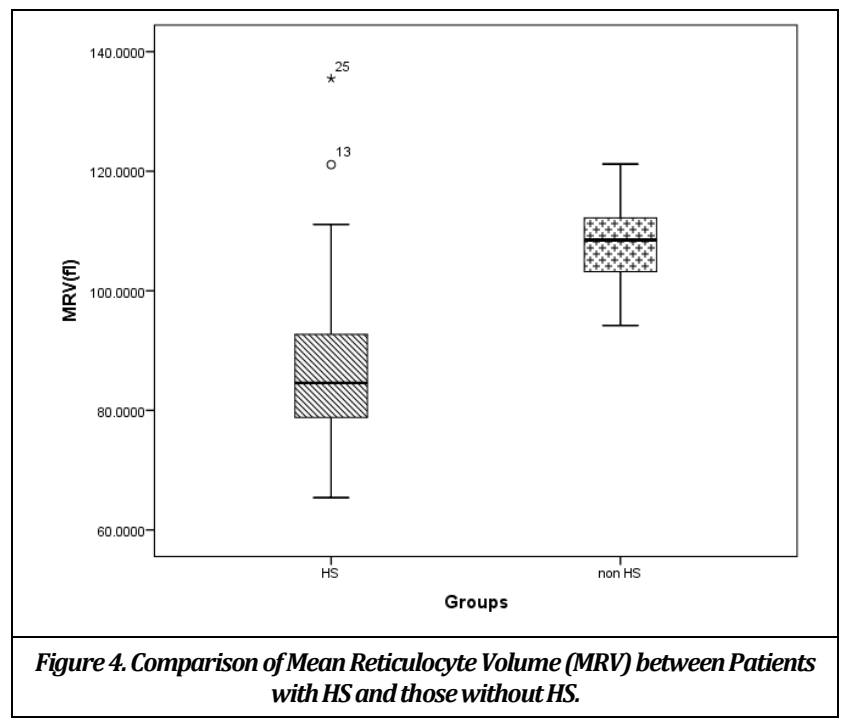

Mean Sphered Cell Volume (MSCV) was available for only 2 cases which was 65.8 and $70.0 \mathrm{fl}$ respectively. In both these cases MSCV was found to be less than MCV. RBC size factor which is calculated as $\sqrt{M C V x}$ MRV was obtained in both patients with HS and in normal healthy controls. The mean value in patients was 87.32 and that for normal controls was 97.49. This difference in mean value of RBC size factor across the two groups were found to be statistically significant $(\mathrm{p}<0.001)$. Cut-off values derived for MCHC was $\geq 33.5$. The reticulocyte parameters such as MRV, IRF and Retic\#/IRF had cut-off values of $\leq 98.8 \mathrm{fl}, \geq 0.33$ and $\geq 0.28$ respectively. (Table 7).

\section{DISCUSSION}

A retrospective study was conducted to assess the usefulness of reticulocyte parameters for the diagnosis of hereditary spherocytosis. The reticulocyte parameters were obtained by Beckman Coulter LH 750 analyzer. It has already been hypothesized that the automated reticulocyte parameters could be useful in the screening of HS especially if small dehydrated reticulocytes are demonstrated; because the surface area loss in HS is not only due to microvesiculation or spleen conditioning, but is also a phenomenon that is already present at the reticulocyte stage. 5

A total of 25 cases of hereditary spherocytosis were analyzed. These were obtained based on the initial screening by the presence of spherocytes on peripheral smears which was suggestive of HS, along with an increased osmotic fragility test. The mean age of patients was 22 years with an age range of 1- 51 years in the present study. A study done by Nair et al $^{6}$ also had a similar age distribution which ranged between 0 to 50 years.

The various parameters studied included Hemoglobin concentration, Red blood cell count, Mean corpuscular volume (MCV), Mean corpuscular Hemoglobin Concentration (MCHC) and Red cell distribution width (RDW). The hypothesis that the reticulocyte indices would be useful for the screening of HS was tested with special emphasis laid on reticulocyte parameters such as retic percentage, absolute reticulocyte count, Immature reticulocyte fraction, mean reticulocyte volume and mean Sphered cell volume (MSCV).
The mean corpuscular haemoglobin concentration is greater than $360 \mathrm{~g} / \mathrm{L}$ in half of patients because of cellular dehydration ${ }^{2}$. Combination of the mean corpuscular haemoglobin concentration with the red blood cell distribution width can lead to a specificity of nearly $100 \%{ }^{2}$ Our study showed a significant statistical difference in retic percentage and Red cell distribution width across the study group and the control group. Both these parameters were significantly higher in patients with HS when compared to the normal controls.

Lazarova et al, 7 in their study had a median reticulocyte percentage of $19.2 \%$ and RDW of $17 \%$ in patients with HS. Median values of reticulocyte percentage and RDW in our study was $12.7 \%$ and $19 \%$ respectively. The median value of MCHC obtained by Lazarova E et al was $33.8 \mathrm{~g} / \mathrm{dl}$ which was slightly lower when compared to that obtained in the present study which was $34.9 \mathrm{~g} / \mathrm{dl}$.

From the different reticulocyte parameters which were analyzed, area under the curve was calculated for IRF, MRV and Retic\#/IRF which was 0.867, 0.865 and 0.931 respectively. Ciepiela 0 et $\mathrm{al}^{8}{ }^{8}$ in their study derived AUC for MRV which was 0.963 with a sensitivity of $87.66 \%$ and specificity of $94.12 \%$, at a cut off value of $91.15 \mathrm{fl}$. AUC for reticulocyte parameters were also calculated by Lazarova $\mathrm{E}$ et al. The AUC for MRV, IRF and Retic\#/IRF were $0.98,0.50$ and 0.95 respectively from which they derived cut off values of $<96.7,>0.19$ and $>1.5$ respectively. The cut- off values derived for MRV was comparable to that obtained by Lazarova $E$ et al. However, the IRF cut -off value in the present study was higher as compared to the study done by Lazarova $\mathrm{E}$ et al. Our study found that these parameters were good predictors of the disease with the given cut off values.

Nair SC et $\mathrm{al}^{6}$ in their study of reticulocyte parameters in HS, observed that nearly $56 \%$ of the HS cases had an MRV which was less than MCV when compared to the normal cases which had MRV greater than MCV. A similar finding was also observed by Da Costa et al. who suggested that the reticulocytes of HS were smaller when compared to those of other hemolytic anemias. Our study also observed that among the HS patients $63.6 \%$ of the cases had MRV less than that of MCV whereas all the normal subjects had MRV greater than MCV which was concordant to the previously mentioned studies. Yet another parameter that we analyzed was the RBC size factor. RBC size factor is calculated as $\sqrt{M C V x}$ MRV. This parameter was compared among patients and controls the HS patients had a lower value compared to the normal controls which was also statistically significant.

Similar observations were made by Ciepiela 0 et $\mathrm{al}^{8}$ in their study in which the RBC size factor was found to be low in patients with HS. Since the calculation of Rsf is based MSCV is another parameter which has been studied by few authors and have been proved to have high sensitivity and specificity in screening of HS. Broseus et al, ${ }^{9}$ Lazarova E et al, ${ }^{7}$ Nair SC et $\mathrm{al}^{6}$, Chiron $\mathrm{M}$ et $\mathrm{al}^{10}$ have all observed that (MCV- MSCV) was higher in HS patients. In the present study, MSCV was available only in 2 cases. However, in both these cases, MSCV was less than MCV. MSCV was the parameter that was suggested to be the most reliable of all red blood cell indices in identifying HS patients. It is the erythrocyte volume under hypo-osmotic condition. The obtained Mean Sphered Corpuscular Volume (MSCV) in regular red blood cells is higher than the Mean Corpuscular Volume (MCV) measured 
under iso-osmotic conditions. In hereditary spherocytosis, on the contrary, MSCV of spherocytes was lower than MCV due to the decreased ratio of cell surface area to cell volume, that causes these spherocytes to haemolyse and hence give a lower value of MSCV.6,7,9

\section{CONCLUSIONS}

Though reticulocyte indices are now available in most of the hematology analyzers, their use in clinical practice is limited. So, we emphasize the usefulness of these parameters and propose a new diagnostic tool using these parameters for the diagnosis of hereditary spherocytosis. We recommend the following cut off values of reticulocyte parameters for the diagnosis of HS. MRV<98.8, IRF $>0.33$ and retic\#/IRF $>0.28$. However, more studies are needed to implement retic parameters as a screening tool for the diagnosis of Hereditary Spherocytosis. The advantages of using these parameters are it cost effectiveness, non-invasive nature, and easy availability in most of the hematology analyzers.

\section{Limitations}

In the present study, we could not obtain the data of MSCV for most of our patients as it was not available.

\section{REFERENCES}

[1] Mariani M, Barcellini W, Vercellati C, et al. Clinical and hematological features of 300 patients affected by hereditary spherocytosis grouped according to the type of the membrane protein defect. Hematologica 2008;93(9):1310-7.
[2] Perrotta S, Gallagher PG, Mohandas N. Hereditary Spherocytosis. Lancet 2008;372(9647):1411-26.

[3] Bolton-Maggs PH, Langer JC, Iolascon A, et al. Guidelines for the diagnosis and management of hereditary spherocytosis-2011 update. British Journal of Hematology 2012;156(1):37-49.

[4] Bianchi P, Fermo E, Vercellati C, et al. Diagnostic power of laboratory tests for hereditary spherocytosis: a comparison study in 150 patients grouped according to molecular and clinical characteristics. Hematologica 2012;97(4):516-23.

[5] De Costa LD, Mohandas N, Sorette M, et al. Temporal differences in membrane loss lead to distinct reticulocyte features in hereditary spherocytosis and in immune hemolytic anemia. Blood 2001;98(10):2894-9.

[6] Nair SC, Arora N, Jain S, et al. Mean reticulocyte volume enhances the utility of red cell mean sphered cell volume in differentiating peripheral blood spherocytes of hereditary spherocytosis from other causes. Indian J Pathol Microbiol 2015;58(3):307-9.

[7] Lazarova E, Pradier O, Cotton F, et al. Automated reticulocyte parameters for hereditary spherocytosis screening. Ann Hematol 2010;93:1809-18.

[8] Ciepiela 0, Adamowicz-Salach A, Ragowska A, et al. Usefulness of reticulocyte parameters for diagnosis of hereditary spherocytosis in children. Indian J of Hematol and Blood Transfus 2017;33(2):239-47.

[9] Broseus J, Visomblain B, Guy J, et al. Evaluation of mean sphered corpuscular volume for predicting hereditary spherocytosis. Int J Lab Hematol 2010;32(5):519-23.

[10] Chiron M, Cynober T, Mielot F, et al. The GEN.S: a fortuitous finding of a routine screening test for hereditary spherocytosis. Hematol Cell Ther 1999;41(3):113-6. 